“C2019 IEEE. Personal use of this material is permitted. Permission from IEEE must be obtained for all other uses, in any current or future media, including reprinting/republishing this material for advertising or promotional purposes, creating new collective works, for resale or redistribution to servers or lists, or reuse of any copyrighted component of this work in other works." 


\title{
Comparative Study of Axial Flux Vernier Machine with SMC Cores for Electric Vehicle Application
}

\author{
Chengcheng Liu ${ }^{1}$, Youhua Wang ${ }^{*}$, Gang Lei $^{2}$, Youguang Guo ${ }^{2}$ and Jianguo Zhu ${ }^{2}$ \\ ${ }^{1}$ State Key Laboratory of Reliability and Intelligence of Electrical Equipment (School of Electrical Engineering, \\ Hebei University of Technology), Tianjin, China \\ ${ }^{2}$ School of Electrical and Data Engineering, University of Technology, Sydney, Australia
}

Email: 2016020@hebut.edu.cn

\begin{abstract}
Two high performance axial flux Vernier machines (AFVM) with soft magnetic composite (SMC) cores and one of that with silicon steel cores were proposed and quantitative studied in this paper. By using 3D finite element method (FEM), the torque ability and power factor of above three machines have been optimized. With the combination of spoke magnet rotor structure and 3-D stator structure, the proposed AFVM1 can own the benefit of much higher power factor when compared with other machines, and AFVM2 with double rotor configuration and 3D stator structure can have much higher torque ability comparing with the AFVM with silicon steel cores. It can be seen that with the adoption of SMC material the performance of traditional AFVM has been improved greatly.
\end{abstract}

\section{INTRODUCTION}

Permanent magnet Vernier machines (PMVM) have attracted great of interests from researchers for its merits of very simple structure and high torque ability. Its working principles has been revealed as both the fundamental and harmonic magnetic field contributes together to produce the electromagnetic torque. Due to its advantages of high torque ability, it has been a good candidate machine for the electric vehicle and wind generate applications. In the last decades, many new Vernier machine were proposed and prototyped $^{[1],[2]}$, including the axial flux Vernier permanent magnet machine, spoke magnet rotor machine and etc..

In this paper, two new high performance axial flux Vernier machine (AFVM) with the soft magnetic composite (SMC) cores and one of that with traditional silicon steel cores were proposed. As the adoption of 3D stator structure the new AFVM can output higher torque density compared with the AFVM with silicon steels. By using the analytical method and 3D finite element method (FEM) the main dimension of AFVM have been deduced and optimized and their performance were obtained and quantitatively compared with the traditional interior PM machine.

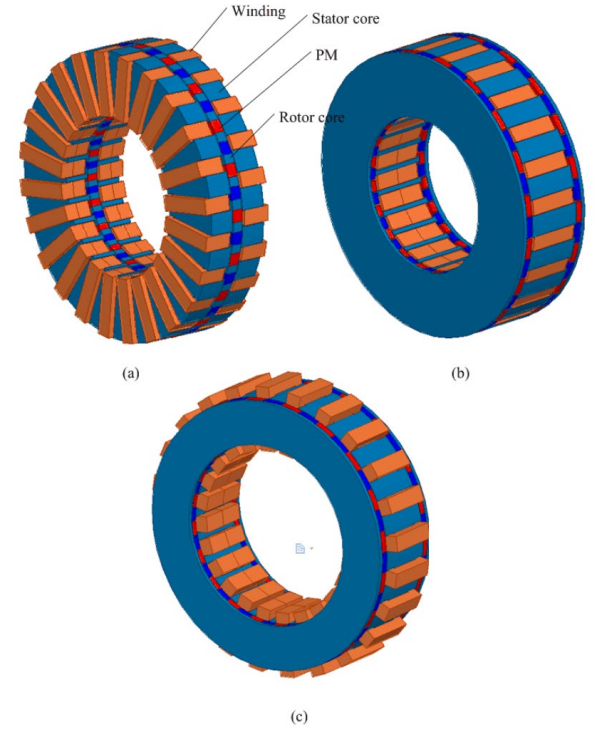

Fig. 1. Topology of AFVM, (a) AFVM1, (b) AFVM2, and (c) AFVM3

\section{Topology OF AFVM}

Fig. 1 illustrates the main magnetic topology of proposed AFVMs. As shown AFVM1 has one spoke magnet rotor in between of two stator cores. Both the rotor cores and stator cores are manufactured by using the SMC materials. Compared with the conventional AFVM, the main difference is that the proposed AFVM has the extended stator teeth in the radial direction thus more magnetic flux can be produced for each winding. The PM material is the ferrite magnet material and the PMs are magnetized along the circumferential direction. The windings are wounded on the stator yoke.

Fig. 1(b) illustrates the main magnetic topology of AFVM2, it has 3D magnetic flux stator core and toroidal winding wounded on stator yoke and with two outer side surface mounted PM rotor cores and one stator cores in between and the PM is magnetized along the axial direction. Fig. 1(c) shows the main magnetic topology of AFVM3, it is quite similar with that of AFVM2, however its stator core are made by using the silicon steels and thus its stator core can not be extended to have higher magntic load.

For Vernier machine, the relationship between the stator slot number, PM pole pairs and armature winding pole pair number is given by, 


$$
P_{w}=\left|P_{m} \pm P_{s}\right|
$$

where $\mathrm{Pw}$ is the armature winding pole pair number, $\mathrm{Pm}$ the PM pole pair number and Ps the stator slot number. When the above relationship is met, then the Vernier effect will be produced. And a series of flux density space harmonics will be produced, and it can be expressed by,

$$
\begin{aligned}
P_{m, n}=\left|m P_{m} \pm n P_{s}\right| & m=1,3,5, \ldots, \infty \\
& n=0, \pm 1, \pm 2, \pm 3, \ldots, \pm \infty
\end{aligned}
$$

The harmonics order of Pm-Ps plays an important role to boost the back EMF while in the conventional PM machine only one flux works to produce the torque.

\section{MAGNETIC FIELD ANALYSIS}

Considering that the permeability of SMC material is quite low and the stator structure is very complex, the $3 \mathrm{D}$ FEM is used for the magnetic field and parameter analysis. By using the 3D FEM method, the no load magnetic flux density distributed on the stator core of AFVMs can be obtained. As shown in Fig. 2, it can be seen that with the adoption of high co-energy magnet, the tips of stator teeth are saturated. Due to the magnetic modulate principle the stator teeth in the AFVM plays the main role, and magnetic flux density distributed on the stator teeth is quite high, the average flux density approaches to $1.2 \mathrm{~T}$, while the magnetic flux density on the stator yoke is quite lower. However for the loaded magnetic filed analysis, the flux density on the stator yoke will be increased greatly. The PM flux linkage of AFVM versus its rotor position are illustrated in Fig. 3. It can be seen that all the PM flux are near the sinusoidal waveform.



Fig. 2. No load magnetic flux density distribution on stator core of AFVM, (a) AFVM1, (b) AFVM2, (c) AFVM3

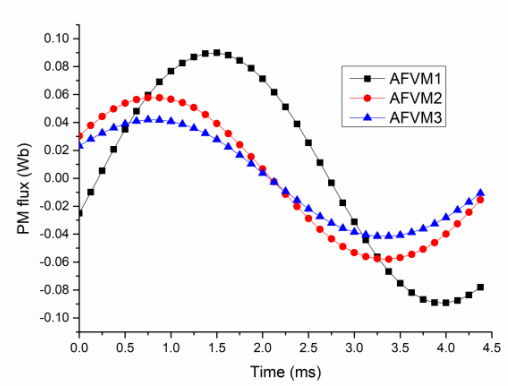

Fig. 3. PM flux linkage of AFVM, (a) AFVM1, (b) AFVM2, (c) AFVM3

\section{DESIGN OPTIMIZATION}

In the design of AFVM, there are main parameters needs to be considered. In this paper, the target machine is the Prius 2004 machine, the main magnetic dimension of these AFVMs are similar to that of Prius 2004, which including the outer radius of $134.5 \mathrm{~mm}$ and axial length of $76 \mathrm{~mm}$. It needs to be noticed that the axial length of developed AFVM only equals to the lamination length of Prius 2004 machine and the end winding length are saved.

In the design of AFVM, considering the minimum length of the SMC cores can not be less than $3 \mathrm{~mm}$, and the parallel stator slot is selected for improving the slot fill factor, the inner stator radius has a close relationship with the stator slot width. And Considering the stator teeth in the AFVM needs to play the magnetic modulate role, thus the width of the stator teeth can not be very high, in this paper the minimum length of SMC cores are designed to equal $4 \mathrm{~mm}$ and then the width of the stator teeth has been determined. Then the stator slot height will be the first important parameter for the AFVM since it determines the balance of the magnetic load and the electric load, when the stator slot height increases the electric load increases and the magnetic load decreases.

In the AFVM, the main torque is the PM torque and the reluctance torque can be neglected since the reluctance torque is very low. For torque calculation the d-axis current equals zero is used. By using the 3D FEM, the torque ability of AFVM1 versus its stator slot height is shown in Fig. 4. It can be seen that when the stator slot height equals about 6 $\mathrm{mm}$, then its torque can be maximized. Another important parameter is the power factor, for the traditional AFVM the power factor is very low, thus during the design optimization process the power factor needs to be refered as well. In the traditional PM machine design the improving the electric load will decrease the power factor, it can be seen in Fig. 5, the power factor of AFVM1 decreases with the stator slot height increases. For the overall consideration, the stator slot height of $6 \mathrm{~mm}$ is determined.

As the spoke structure is used in the AFVM1, the ratio of thickness of PM and thickness of rotor parts needs to be optimized. Increasing the PM thickness will make the AFVM1 with higher magnetic load, which can be good for improving the torque ability and power factor. However, when the thickness is over to a determined value then the rotor part will be saturate and the effect magnetic flux density produced on the stator part will be decreased. As 
shown in Fig. 6 and Fig. 7, the maximum torque of AFVM1 can be achieved when its PM thickness equals $5 \mathrm{~mm}$ and the maximum power factor has been achieved when the PM thickness equals $5 \mathrm{~mm}$.

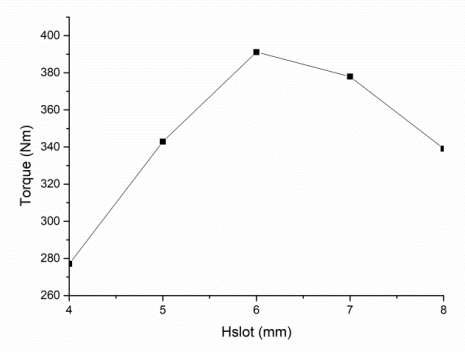

Fig. 4. Torque versus the height of stator slot in AFVM1

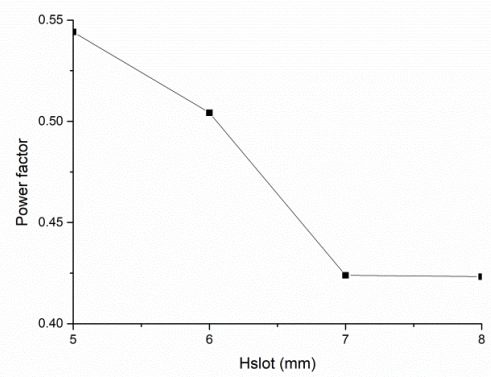

Fig. 5. Power factor versus the height of stator slot in AFVM1



Fig. 6. Torque versus the height of thickness of PM in AFVM1



Fig. 7. Power factor versus the height of thickness of PM in AFVM1

For AFVM2, the maximum torque ability can be achieved when the stator slot height equals $10 \mathrm{~mm}$, and its power factor decreases with the stator slot height increases, for the overall consideration the optimized stator slot height is determined to equal $10 \mathrm{~mm}$, as shown in Fig. 8 and Fig. 9. For the PM thickness optimization in AFVM2, the difference against the AFVM1 is that the surface mounted PM structure is selected, and both the torque ability and power factor increases with the thickness increases as shown in Fig. 10 and Fig. 11, however for the cost consideration, the thickness of PM can not be very high, and thus the PM thickness equals $4.5 \mathrm{~mm}$ is selected. For the AFVM3, since the main structure of AFVM3 is quite similar to that of the AFVM2, and for the fair comparison, the outer radius of the AFVM3 needs to add both the stator core and the length of the stator winding in the radial direction, and thus only the height of the stator winding has been optimized. Some other parameters in the AFVMs has been optimized as well, and final main parameters and dimensions of these AFVMs have been tabulated in Table1.



Fig. 8. Torque versus the height of stator slot in AFVM2

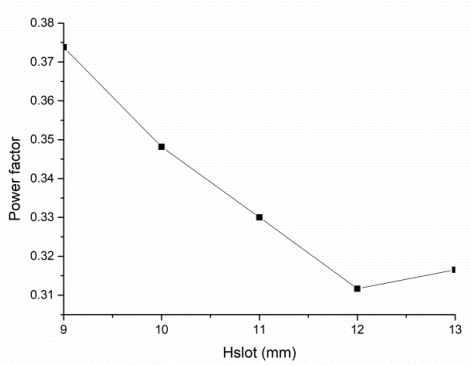

Fig. 9. Power factor versus the height of stator slot in AFVM2



Fig. 10. Torque versus the height of Thickness of PM in AFVM2 


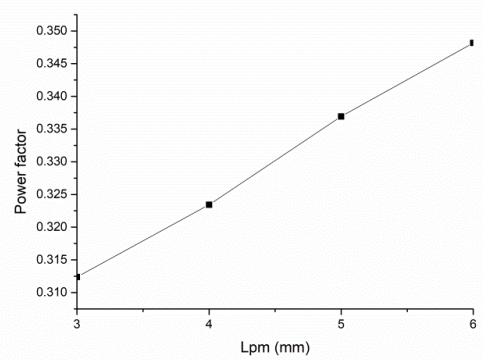

Fig. 10. Power factor versus the height of Thickness of PM in AFVM2

Table I Main Dimension of Proposed AFVM1 and AFVM2

\begin{tabular}{|c|c|c|c|c|}
\hline Parameter name & AFVM1 & AFVM2 & AFVM3 & Unit \\
\hline Axial length & 76 & 76 & 76 & $\mathrm{~mm}$ \\
\hline Stator outer radius & 134.5 & 134.5 & 134.5 & $\mathrm{~mm}$ \\
\hline Stator inner radius & 74 & 74 & 74 & $\mathrm{~mm}$ \\
\hline Air gap length & 1 & 1 & 1 & $\mathrm{~mm}$ \\
\hline Height of stator slot & 6 & 11 & 10 & $\mathrm{~mm}$ \\
\hline Width of stator slot & 15.4 & 15.4 & 15.3 & $\mathrm{~mm}$ \\
\hline $\begin{array}{l}\text { Axial length of stator } \\
\text { core }\end{array}$ & 32 & 54 & 54 & $\mathrm{~mm}$ \\
\hline Thickness of PM & 10 & 6 & 6 & $\mathrm{~mm}$ \\
\hline $\begin{array}{c}\text { Angle of PM near outer } \\
\text { radius }\end{array}$ & 5 & & & deg \\
\hline $\begin{array}{c}\text { Angle of PM near inner } \\
\text { radius }\end{array}$ & 4 & & & deg \\
\hline $\begin{array}{l}\text { Number of winding } \\
\text { turns }\end{array}$ & 10 & 10 & 10 & \\
\hline
\end{tabular}

\section{MAIN PERFoRMANCE COMPARISON}

After the optimization process of AFVMs, the main performance of these AFVMs are compared. In the performance comparison, the torque ability and the power factor are very important. Fig. 11 shows the torque comparison among these AFVMs, it can be seen that the torque ability of the AFVM2 is the highest one and that of AFVM3 is the lowest one. Compared with the AFVM1, there is no any material and space waste in the AFVM2, and thus the torque ability is higher. As for the AFVM3, the utilization of silicon steels let this machine without the 3D magnetic flux path, thus the torque ability is quite lower.

For the power factor comparison, as proved in [], the spoke rotor structure can let the Vernier machine has higher power factor, and this statements can be utilized in the $\mathrm{AFVM}$ as well. It can be seen that the power factor of AFVM1 is much higher than the other AFVMs, when the applied current density is about $8 \mathrm{~A} / \mathrm{mm} 2$, the power factor of AFVM1 is about 0.85 , while that of AFVM2 is only 0.7 and with the current density increases the power factor of the AFVM2 and AFVM3 decreases faster.

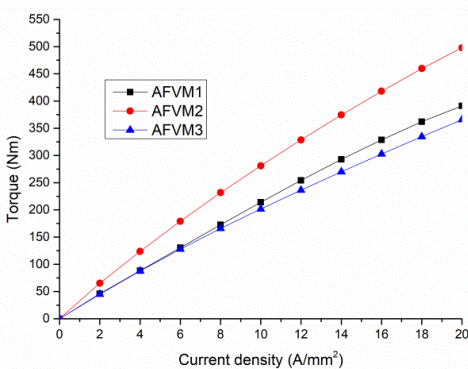

(a)

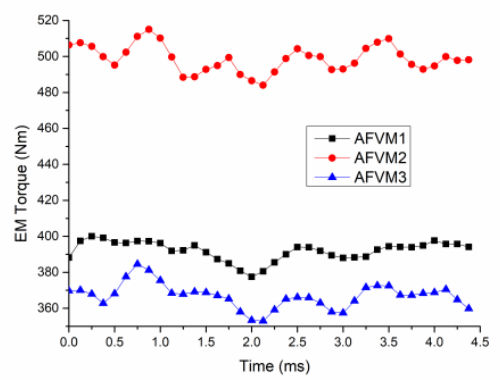

(b)

Fig. 11. Torque comparison, (a) Torque versus current density and (b) Torque waveform



Fig. 12. Power factor comparison

\section{CONCLUSION}

Two new AFVMs with SMC cores were designed, analyzed, optimized and compared in this paper with the benchmark mark machine of a interior PM machine. The results show that the proposed AFVM2 have the highest torque density and moderate power factor, it is a good candidate machine for the EV application.

\section{REFERENCES}

[1] Y. Gao, R. Qu, D. Li, H. Fang, J. Li, and W. Kong, "A novel dualstator Vernier permanent magnet machine," IEEE Trans. Magn., vol. 53, no. 11, Nov. 2017, Art. no. 8110105 .

[2] S. Niu, S. L. Ho, W. N. Fu, and L. L. Wang, "Quantitative comparison of novel Vernier permanent magnet machines," IEEE Trans. Magn., vol. 46, no. 6, pp. 2032-2035, Jun. 2010. 